\title{
Intraoperative Bildgebung in der Beckenchirurgie
}

Peter Hinnerk Richter, Alexander Eickhoff, Florian Gebhard, Konrad Schütze

\section{Einleitung}

Durch die komplexe Anatomie des Beckens ist die konventionelle radiologische 2-D-Darstellung schwer zu analysieren und interpretieren. Die Fraktursituation kann dies zusätzlich erschweren. Für die Planung von Beckeneingriff ist eine präoperative 3-D-Bildgebung mittels Computertomografie (CT) heutzutage unausweichlich. Die Einführung mobiler 3-D-C-Bögen ermöglicht es dem Chirurgen, eine intraoperative 3-dimensionale Kontrolle der Reposition und Osteosynthesenlage durchzuführen. Hierbei dreht sich der C-Bogen in einer isozentrischen Rotation um das Operationsgebiet. Durch die Kombination mit Navigationssystemen konnte die Genauigkeit der Schraubenlagen gerade im Bereich des hinteren Beckenrings verbessert werden [1]. Die Bildqualität der 3-D-CBögen ist jedoch mit einem CT nicht zu vergleichen und ist für anatomische Regionen wie z. B. den zervikothorakalen Übergang oder das Becken nur eingeschränkt geeignet. Zudem ist das Darstellungsvolumen aufgrund einer kleinen Detektorgröße relativ gering.

Der rasante technische Fortschritt der letzten Jahre führte auch zu einer Entwicklung moderner intraoperativer Bildgebungssysteme. Diese werden entweder als fixierte oder mobile Systeme angeboten und führten zu einer deutlichen Verbesserung der Bildqualität und Vergrößerung des Bilddurchmessers [2]. Dies kann gerade in der Beckenchirurgie eine entscheidende Hilfe sein.

\section{Fortschritte der intraoperativen Bildgebung}

\section{Flachdetektor-C-Bögen}

Die Flachdetektortechnologie führt zu einer Verbesserung der Bildqualität mit einer begleitenden Vergrößerung des Bilddurchmessers. Zudem kommt es zu einer Reduzierung der angewendeten Dosis [3,4]. Des Weiteren führen eine größere Grauskala und eine höhere Pixelzahl zu einer Optimierung der Bildqualität. Bei konventionellen Bildverstärkern kann es aufgrund von Magnetfeldern zu Verzerrungen im Bereich des Bildrandes kommen. Dies tritt bei Flachdetektor-C-Bögen nicht mehr auf [2].
Die Flachdetektortechnologie war zu Beginn nur bei fixierten Bildgebungssystemen und C-Bögen verfügbar. Seit einigen Jahren stehen auch mobile Flachdetektor-CBögen zur Verfügung. Die Dosiseffizienz ist nicht der einzige Grund, warum sie besonders für unfallchirurgische Operationssäle geeignet sind [5]. Die Vergrößerung des Detektors ist besonders in der Beckenchirurgie eine entscheidende Verbesserung. Hier können komplexe Frakturen vollständig abgebildet werden. Zudem kann intraoperativ die verletzte mit der gesunden Seite verglichen werden. Des Weiteren führt der Einbau von Flachdetektoren in mobilen C-Bögen zu einem erhöhten Abstand zwischen Röntgenquelle und Detektor. Dadurch werden entscheidende Zentimeter gewonnen, um überstehende Führungsdrähte oder Repositionszangen problemlos im Operationsgebiet zu belassen. Zusätzlich kann bei der Akquirierung von Inlet- und Outletbildern bei adipösen Patienten ein vergrößerter Fokus-Bildempfänger-Abstand eine entscheidende Hilfe sein. Manche Systeme verfügen zudem über die Möglichkeit einer asymmetrischen Kollimation. Hierdurch können Instrumente oder Metallteile des OP-Tisches ausgeblendet werden und zusätzlich zu einer Verbesserung der Bildqualität beitragen [2].

Die Flachdetektortechnologie ist nicht nur auf die 2-DBildgebung beschränkt. Schlechte Knochenqualität, Überlagerung durch Darmgase oder adipöse Weichteilverhältnisse können eine ungenügende intraoperative fluoroskopische Darstellung bedingen. Hierdurch kann die Beurteilung der Reposition oder Implantatlage erschwert sein [6]. Eine entscheidende Hilfe stellen hierbei 3-D-Flachdetektor-C-Bögen dar. Diese führen ähnlich wie konventionelle 3-D-C-Bögen eine Rotation um das Operationsgebiet durch. Aufgrund des kegelförmigen Röntgenstrahls wird dieser auch als „Cone-Beam-CT“ bezeichnet. Dabei wird eine bestimmte Zahl an Einzelbildern erstellt. Diese werden im Anschluss zu einem 3-D-Datensatz rekonstruiert. Dieser kann i.d.R. als multiplanare Rekonstruktion (MPR) oder auch als 3-D-Volumen (volume rendering technique: VRT) dargestellt werden [7].

Die 3-D-Darstellung führt zu einer unmittelbaren Kontrolle der intraoperativen Ergebnisse. Seit Einführung von minimalinvasiven Operationstechniken am Becken werden die Grenzen zunehmend ausgelotet. Wurden zu Beginn vor allem nicht oder geringgradig dislozierte Sa- 
krumfrakturen mittels geschlossener Reposition und Iliosakralschraube versorgt, so ermöglicht die intraoperative 3-D-Bildgebung minimalinvasive Osteosynthesen auch bei dislozierten Frakturen. Nach Reposition oder Implantation von Osteosynthesematerial kann eine radiologische 3-dimensionale Kontrolle durchgeführt werden. Somit können ungenügende Repositionen oder Implantatfehllagen unmittelbar korrigiert werden [8]. Dadurch können Folgeoperationen und die dabei entstehenden Kosten vermieden werden.

Der O-Arm (Medtronic, USA) ist der prominenteste Vertreter der modernen Cone-Beam-CT-Scanner. Seine Hauptanwendung findet er in der Wirbelsäulenchirurgie [9]. Als mobiler Cone-Beam-CT-Scanner kann er jedoch flächendeckend in der Unfallchirurgie eingesetzt werden $[10,11]$. In der Beckenchirurgie zeigen sich die Vorteile des O-Armes besonders, wenn dieser mit einem Navigationssystem kombiniert wird [9].

Ein weiterer prominenter Vertreter der Cone-Beam-CTScanner ist der Artis zeego (Siemens, Deutschland). Im Gegensatz zu dem O-Arm ist er bodengebunden und kann somit nur in einem Operationssaal eingesetzt werden. Die Kombination aus einem fest installierten Bildgebungssystem in einem Operationssaal wird als HybridOperationssaal bezeichnet ( $\triangleright$ Abb. 1). Beim Artis zeego ist der Flachdetektor an einen Roboterarm fixiert. Er kann somit vollständig autark durch den Operateur gesteuert werden. Außerdem kann jede Position des C-Bogens gespeichert und im Anschluss wieder automatisch angefahren werden [12]. Die Anwendung dieses C-Bogens in der Unfallchirurgie bietet große Vorteile. Durch den großen Detektor kann im Vergleich zu konventionellen 3-D-CBögen mit nur einem 3-D-Scan (in Abhängigkeit der Patientengröße) das gesamte Becken dargestellt werden. Zudem ist die Bildqualität durch die Flachdetektortechnologie deutlich erhöht [13]. Zu Beginn wurde der Artis zeego nur in der Herz- und Gefäßchirurgie als Angiografiesystem verwendet. Durch eine interdisziplinäre Nutzung kann die Auslastung dieses Systems erhöht und die Anschaffungskosten geteilt werden [12].

\section{Intraoperative Computertomografie}

Die intraoperative Computertomografie wird seit vielen Jahren in der Beckenchirurgie eingesetzt. Dabei muss jedoch zwischen einem Computertomografen in einem Operationssaal und minimalinvasiven Eingriffen in einer CTSuite in der Radiologie unterschieden werden. In der ausgelagerten CT-Suite werden bevorzugt Iliosakralschrauben implantiert. Die CT-gestützte Implantation von Iliosakralschrauben zeigte eine hohe Präzision [1]. Allerdings ist diese Form der Implantation ausschließlich für nicht oder geringgradig dislozierte Frakturen des hinteren Beckenrings geeignet. Ein vorhergehender Eingriff (Fixateur externe, Symphysenplatte) bei dislozierten Tile-B-Frakturen kann das Ausmaß der Dislokation verringern, sodass im An-

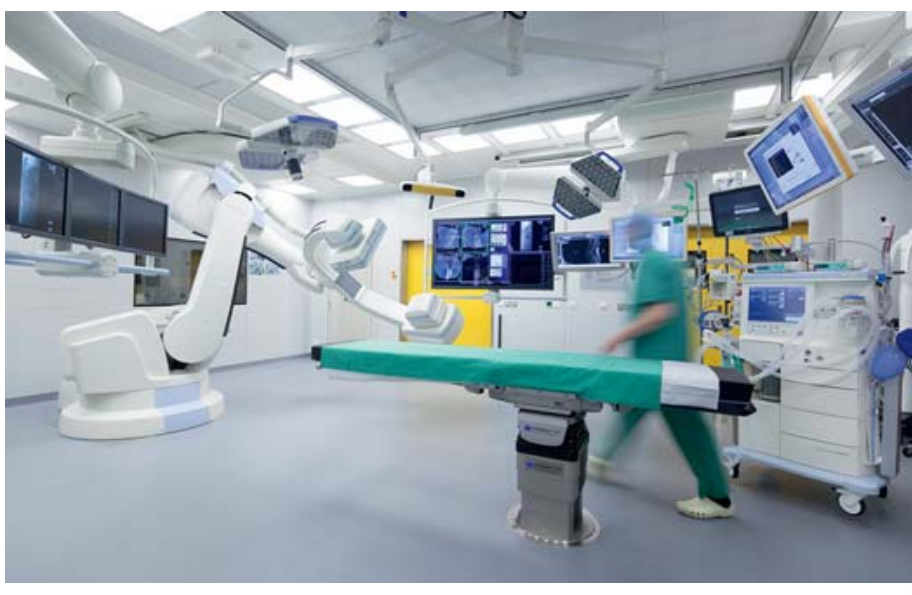

- Abb. 1 Hybrid-OP des Universitätsklinikums Ulm: Links steht der Artis zeego, der digital mit dem Operationstisch und dem Navigationssystem (deckengebunden) verbunden ist.

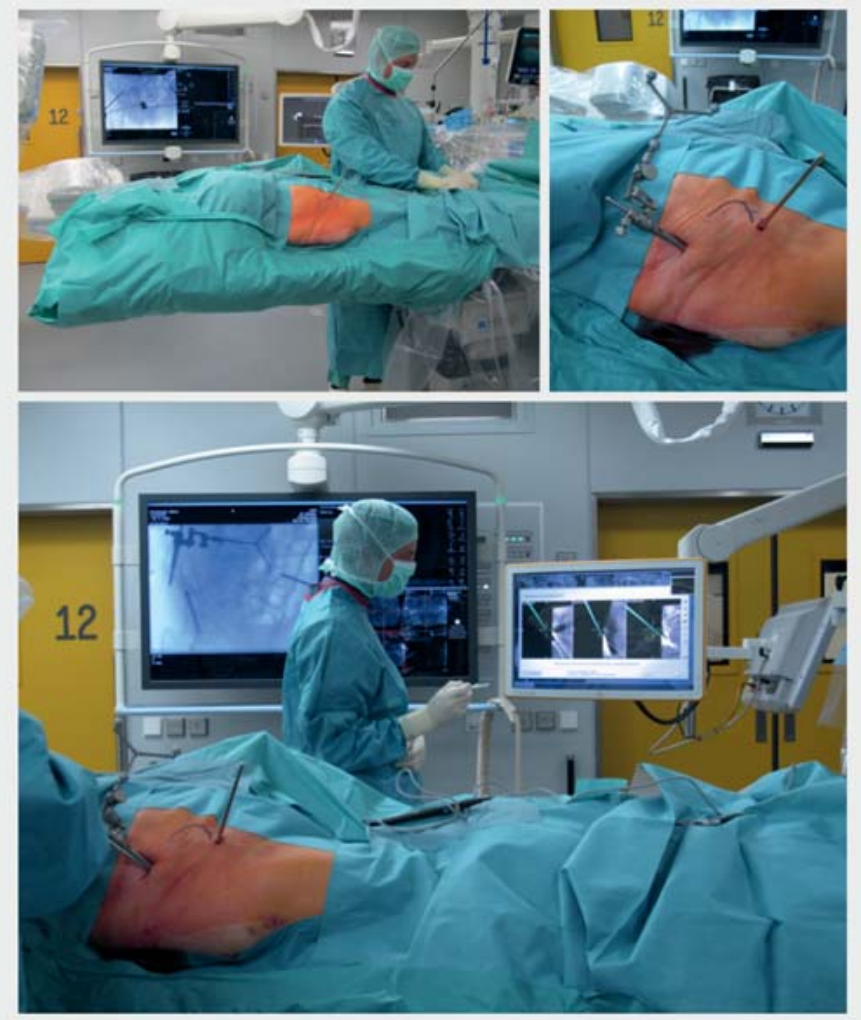

- Abb. 2 Patientenlagerung im Hybrid-OP für eine navigierte Schraubenosteosynthese: Im linken oberen Bild ist die $360^{\circ}$-Abdeckung zu sehen. Diese verhindert, dass während des 3-D-Scans die Tücher auf das sterile Operationsgebiet fallen. In einem 2. Schritt wird die Data Reference Base (DRB) an der Beckenschaufel fixiert (rechtes oberes Bild). Das untere Bild zeigt die Verifikation des 3-D-Scans im Navigationssystem mit dem Pointer. 

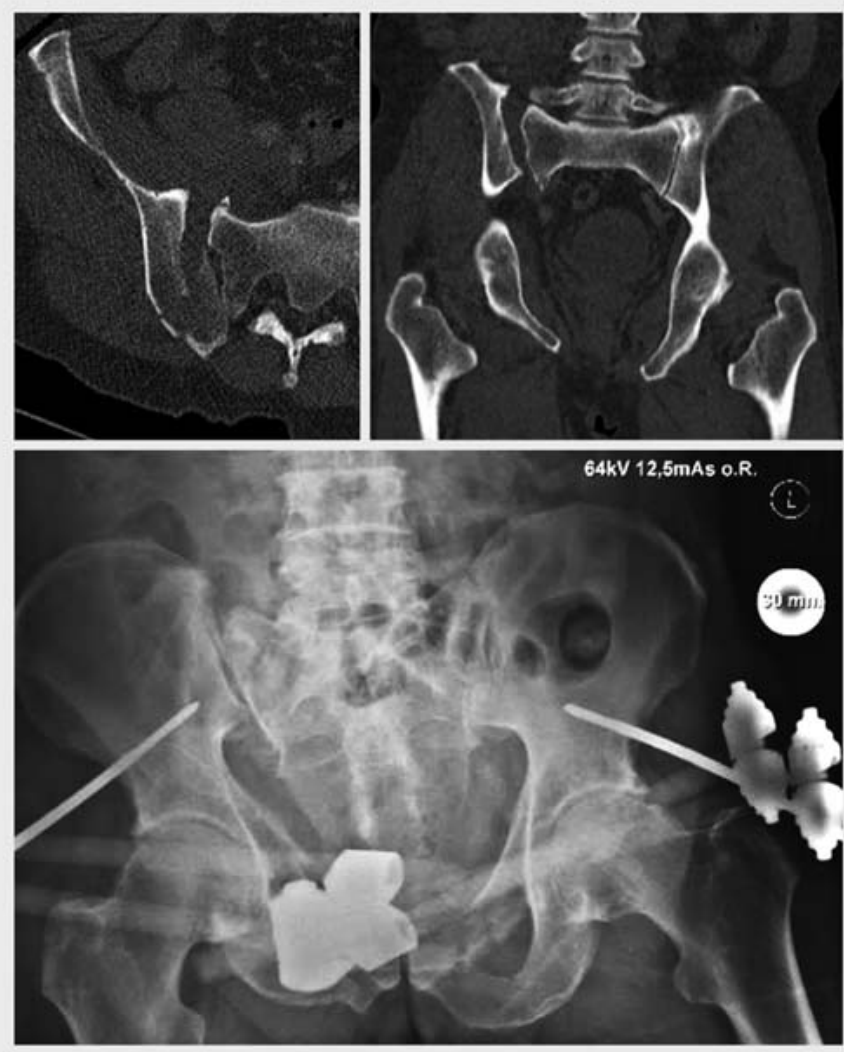

- Abb. 3 Typ-C-Beckenringfraktur: Hier wurde im Rahmen der Primärversorgung ein Fixateur externe angelegt.

schluss eine CT-gestützte Schraubenimplantation im hinteren Beckenring durchgeführt werden kann [14].

Seit einigen Jahren werden vermehrt CT-Scanner in Operationssäle integriert. Fixierte Systeme werden meist auf einem Schienensystem (sliding gantry) fixiert und können somit im Operationssaal bewegt werden. Der CT kann zusätzlich mit einem Navigationssystem kombiniert werden, um eine hohe Präzision zu ermöglichen [15]. Seit Kurzem stehen auch mobile CT-Scanner zur Verfügung $[16,17]$. Dadurch ist der Operateur nicht mehr auf einen festen Operationssaal beschränkt. Allerdings gibt es auch hierfür Einschränkungen. Die mobilen CT sind fest mit einem Operationstisch verbunden und können nicht in Sälen mit vorinstalliertem Tisch eingesetzt werden. Außerdem sollte ausreichend Platz im Operationssaal zur Verfügung stehen, um den CT während der Operation aus dem Operationsgebiet zu schwenken [17]. Für intraoperative fluoroskopische Kontrollen wird im Gegensatz zu Cone-Beam-CT-Scannern ein zusätzlicher C-Bogen benötigt. Als Beispiel der mobilen CT-Scanner ist hier der Airo (BrainLab, Deutschland) zu nennen. Dieser ist automatisch mit einem Navigationssystem verbunden. Erste Studien im Bereich der Wirbelsäule zeigten vielversprechende Ergebnisse [17, 18].

\section{Navigation}

Die Implantation von Iliosakralschrauben ist das Verfahren der Wahl zur Stabilisierung von Frakturen und Dislokationen im Bereich des hinteren Beckenrings. Die fluoroskopisch gestützte Implantationstechnik geht jedoch mit einer hohen Rate an Fehllagen einher $[19,20]$. Durch die Einführung der intraoperativen Navigation konnte die Präzision dieser Schrauben verbessert werden [1]. Die Entwicklung der 3-D-Bildwandler führte zu einer weiten Verbreitung der 3-D-Navigation in Operationssälen. Dies resultierte nicht nur in einer verbesserten Genauigkeit der Schraubenlage, sondern ermöglichte es dem Chirurgen, die Schraubenlage im OP zu kontrollieren [21]. Auch bei Azetabulumfrakturen konnte eine verbesserte Genauigkeit der minimalinvasiven Schraubenosteosynthese gesehen werden [22]. Durch die Anwendung von ConeBeam-CT-Scannern im OP konnte die Genauigkeit weiter erhöht werden. Zudem ermöglicht das erhöhte Visualisierungsvolumen die Implantation von transiliakal-transsakralen Schrauben [13]. Im gleichen Scan kann die gebrochene Seite mit der gesunden Seite verglichen werden.

Ein weiterer Vorteil der intraoperativen Navigation ist die Reduktion der Strahlung. Kraus et al. konnten zeigen, dass die Anwendung eines Navigationssystems bei der Implantation von Iliosakralschrauben zu einer deutlichen Reduktion der intraoperativen Strahlung führt [23].

\section{Eigene Erfahrungen}

\section{Minimalinvasive Schraubenosteosynthese}

In unserem Setting wird der Patient in Rückenlage auf dem Operationstisch gelagert. Nach einer Stichinzision wird eine Schanz-Schraube im Beckenkamm verankert. Diese sollte ca. 2-3 Querfinger dorsal der Spina iliaca anterior superior erfolgen, um den N. cutaneus femoris lateralis nicht zu verletzen. Danach wird die DRB (data reference base) mit einer Hülse an der Schanz-Schraube befestigt ( $\triangleright$ Abb.2). Im Anschluss wird ein 3-D-Scan durchgeführt. Im Hybrid-Operationssaal dauert dieser nur 5-6 Sekunden. Für diesen Zeitraum verlässt das gesamte Personal aus Strahlenschutzgründen den Operationssaal. Der 3-D-Datensatz wird automatisch an das deckengebundene Navigationssystem gesendet. Nach einem Verifikationsschritt wird ein Offset (virtuelle Instrumentenverlängerung) eingestellt. Dadurch können die Schrauben perkutan geplant werden. Danach kann ein „fine-tuning“ der Schraube durchgeführt werden. Im Anschluss wird eine Inzision im Bereich der zuvor geplanten Schraube durchgeführt. Nach stumpfer Präparation auf den Knochen wird das Offset entfernt. Im Anschluss kann ein Kirschner-Draht (K-Draht) mit einer referenzierten Bohrhülse implantiert werden. Dieser kann mit einem 2. 3-D-Scan kontrolliert werden. Hierbei können Fehlla- 

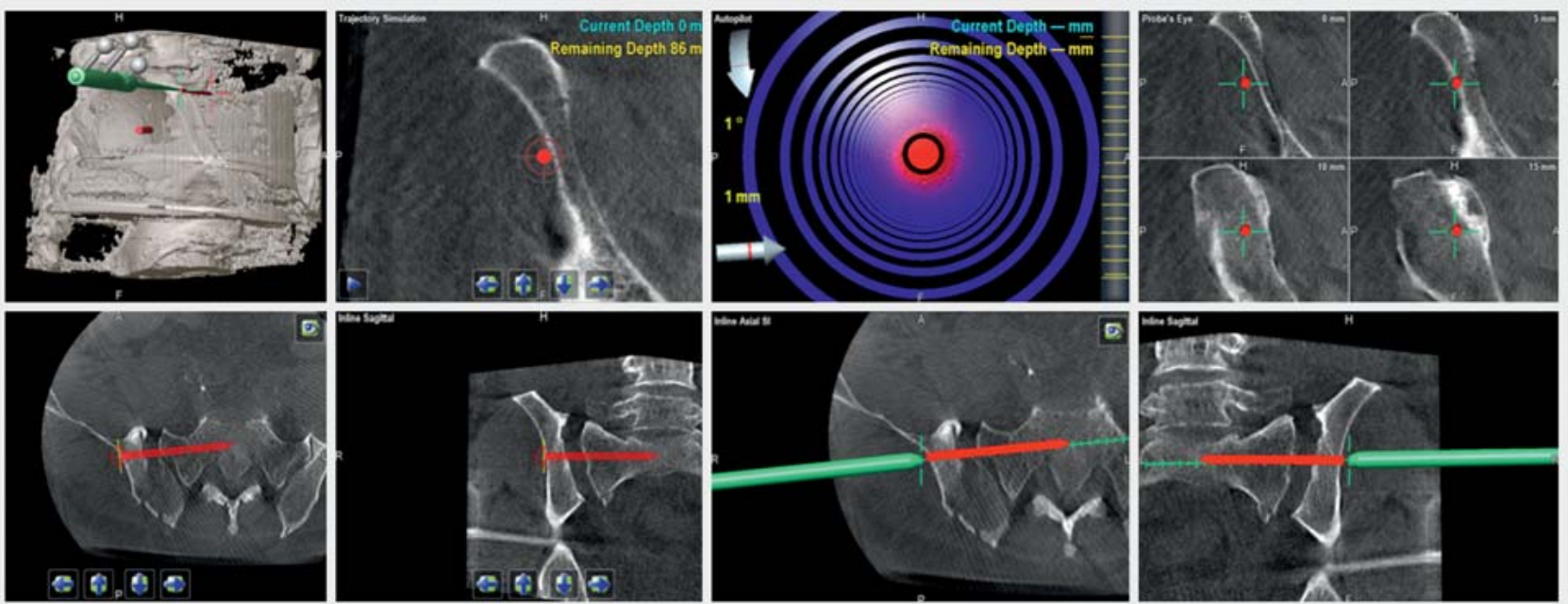

- Abb. 4 Perkutane Planung der Iliosakralschrauben in SI im Navigationssystem (links). Im Anschluss Platzierung des K-Drahtes mittels referenzierter Bohrhülse auf dem Navigationsmonitor (rechts).

gen unmittelbar erkannt und korrigiert werden. Außerdem kann im 3-D-Scan die Schraubenlänge gemessen werden. Nach 3-D-Kontrolle wird die kanülierte Schraube über den Draht implantiert.

Das 1. Fallbeispiel zeigt eine Typ-C-Beckenringfraktur. Nach initialer Fixierung mittels Fixateur externe ( $\bullet$ Abb. $\mathbf{3}$ ) wurde bei noch deutlicher Dislokation die Indikation zur minimalinvasiven Versorgung des hinteren Beckenrings gestellt. Initial wurde die geschlossene Reposition der vertikalen Dislokation durch Längszug am Bein durchgeführt. Die horizontale Dislokation konnte nach Implantation von 3 7,3-mm-Schrauben geschlossen werden ( $\boldsymbol{A} \mathbf{A b b} \mathbf{4}$ ). Aufgrund der großen Kräfte, die für die Reposition notwendig sind, wurden beide lliosakralschrauben in SI durch eine Platte eingebracht, um eine Oberflächenvergrößerung zu erreichen. Im Anschluss zeigt sich eine regelrechte Schraubenlage und Reposition der Fraktur ( $\bullet$ Abb. 5).

Das 2. Fallbeispiel zeigt eine unilaterale Insuffizienzfraktur. Diese kann durch das große Visualisiserungsvolumen mittels transiliakal-transsakraler Schraube versorgt werden ( $\bullet$ Abb. 6).

Im 3. Fallbeispiel zeigt sich eine geringgradig dislozierte Azetabulumfraktur bei einliegender Duokopfprothese ( $\mathbf{A}$ Abb. 7). Typischerweise wird dieser Frakturtyp konservativ therapiert. Hierbei muss eine Entlastung für 6 Wochen durchgeführt werden. Da viele ältere Patienten jedoch keine Entlastung durchführen können, resultiert dies häufig in einer Bettruhe von 6 Wochen. Mittels minimalinvasiver Schraubenosteosynthese kann jedoch eine frühe Aufbelastung ermöglicht und längere Bettruhephasen vermieden werden. Das Vorgehen bez. der Naviga-
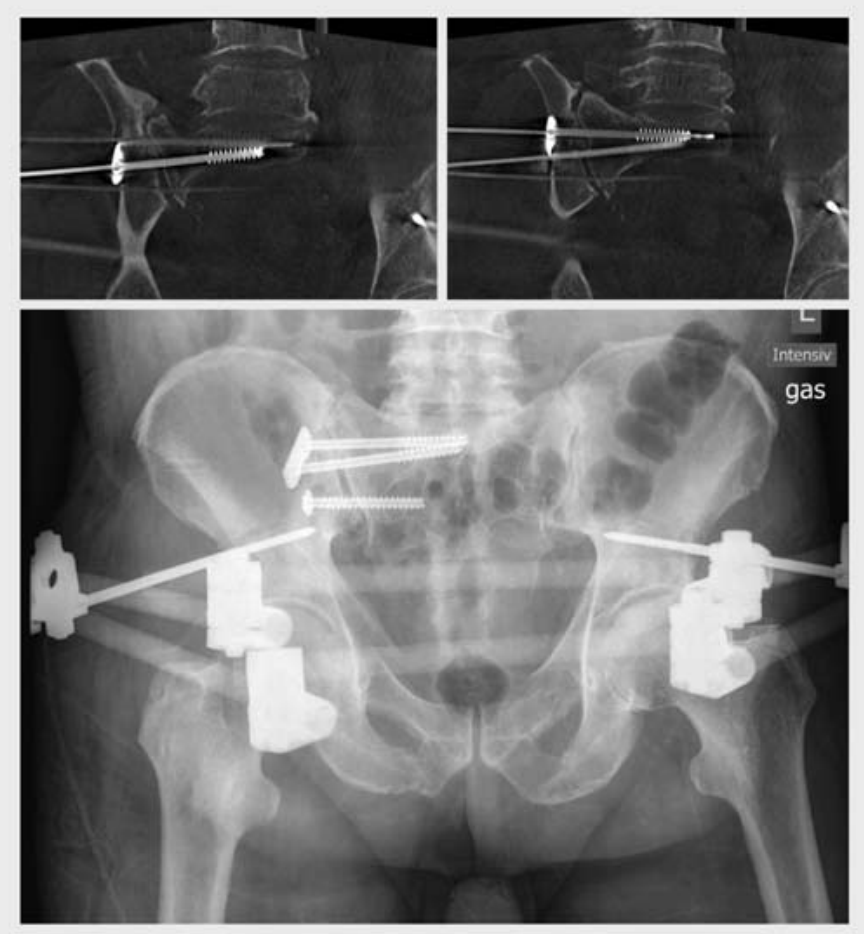

- Abb. 5 Intraoperativer 3-D-Scan und postoperatives Röntgen: Es zeigt sich eine gute Reposition der Typ-C-Fraktur und eine regelrechte Schraubenlage. Zur Oberflächenvergrößerung wurden die S I-Schrauben über eine Platte eingebracht. 

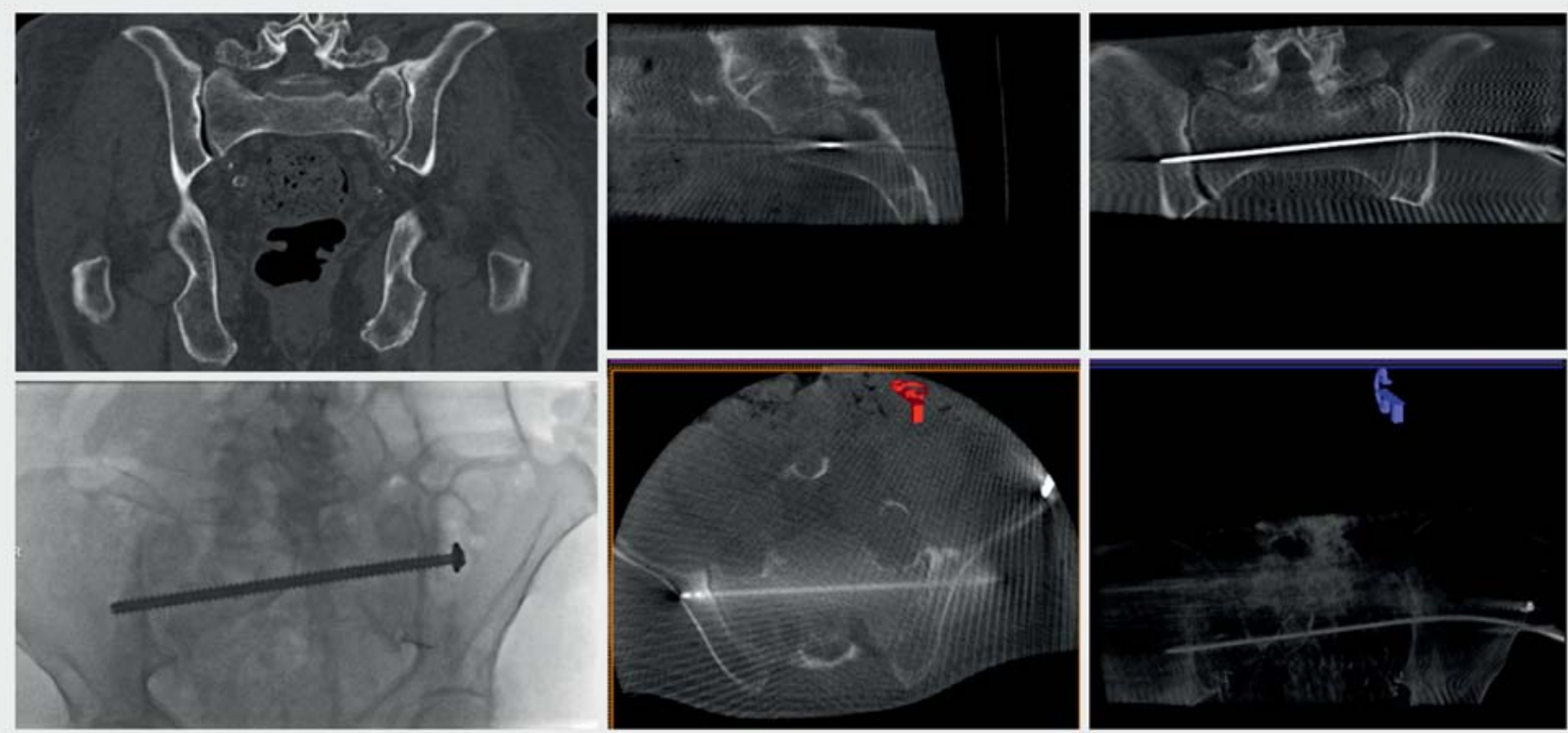

- Abb. 6 Sakruminsuffizienzfraktur links (links oben). Intraoperative 3-D-Kontrolle des K-Drahtes. Hier zeigt sich eine regelrechte Lage ohne Perforation des Neuroforamens oder Spinalkanals (rechts). Postoperative Röntgenkontrolle (links unten).
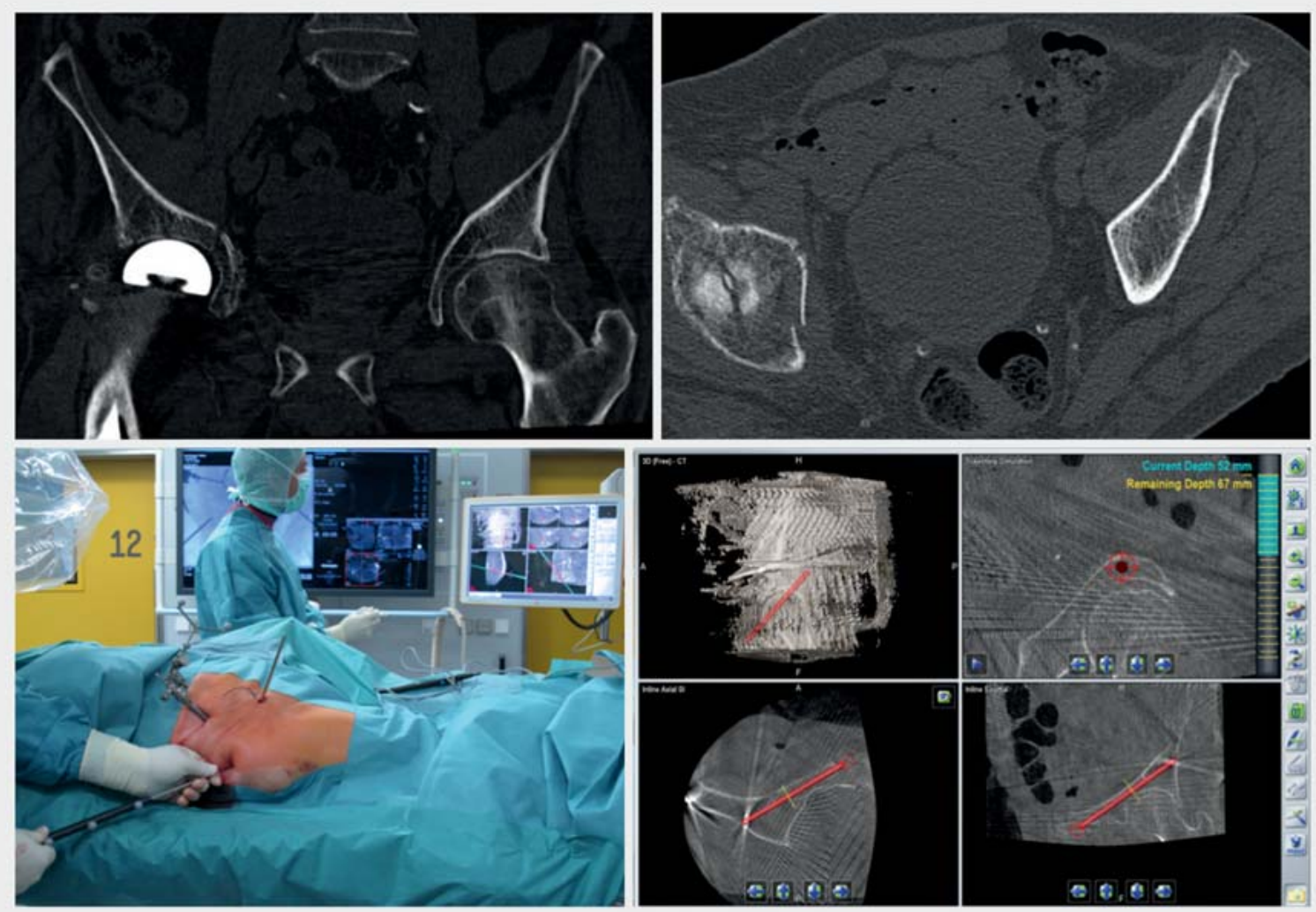

- Abb. 7 Bei geringgradig dislozierter Azetabulumfraktur (oben) wurde die Indikation zur minimalinvasiven navigierten Schraubenosteosynthese gestellt. Lagerung des Patienten im OP und perkutane Planung der vorderen Pfeilerschraube im Navigationssystem (unten). 

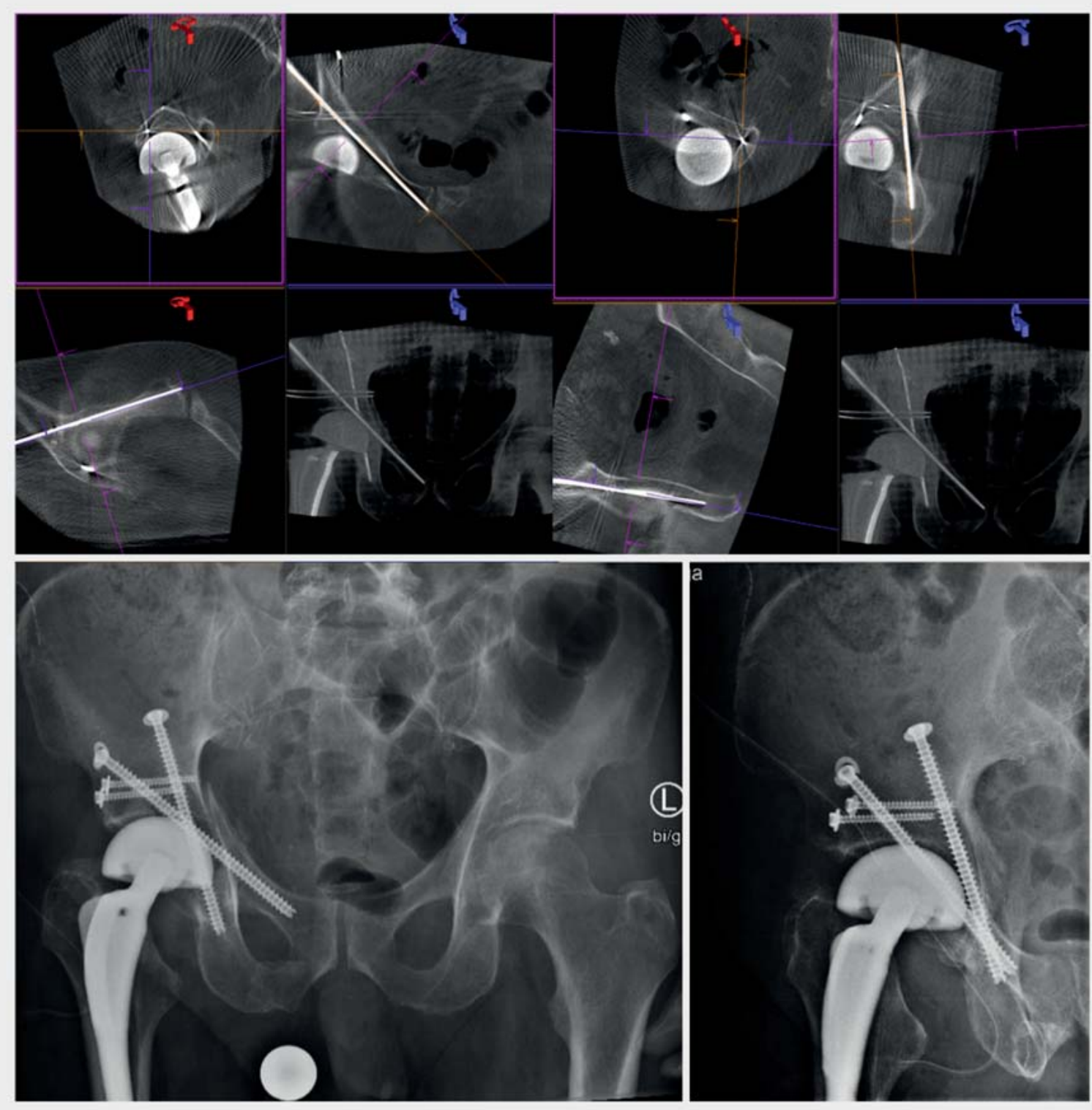

- Abb. 8 Intraoperative 3-D-Kontrolle des K-Drahtes der vorderen (links oben) und hinteren Pfeilerschraube (rechts oben). Trotz einliegender Duokopfprothese kann die K-Draht-Lage problemlos beurteilt werden. Dieses Osteosyntheseverfahren ermöglicht eine frühe Mobilisation bei geriatrischen Patienten.

tion ist vergleichbar mit der Implantation einer Iliosakralschraube ( $\triangleright$ Abb. 8 ).

\section{Schlussfolgerung}

Fortschritte in der intraoperativen Bildgebung ermöglichen die direkte Kontrolle der Reposition und Implantatlage in einer hohen Bildqualität in 2-D und 3-D. Dadurch können Folgeeingriffe vermieden werden. Durch die Kombination mit Navigationssystemen kann die Genauigkeit der Schraubenimplantation gesteigert werden. Durch moderne Bildgebungssysteme können auch kom- plexe Frakturen zunehmend durch minimalinvasive Operationstechniken versorgt werden.

Interessenkonflikt

Prof. Dr. med. F. Gebhard und Dr. med. P. H. Richter halten gelegentlich Vorträge für Siemens Healthcare. Für diese Publikation besteht kein Interessenskonflikt. Für die anderen Autoren besteht keine wirtschaftliche oder persönliche Verbindung. 


\section{Autorinnen/Autoren}

\section{Peter Hinnerk Richter}

Dr. med., Abteilung für Unfall-, Hand-, Plastische- und Wiederherstellungschirurgie, Universitätsklinikum Ulm

\section{Alexander Eickhoff}

Dr. med., Abteilung für Unfall-, Hand-, Plastische- und Wiederherstellungschirurgie, Universitätsklinikum Ulm

\section{Florian Gebhard}

Univ.-Prof. Dr. med., Abteilung für Unfall-, Hand-, Plastischeund Wiederherstellungschirurgie, Universitätsklinikum Ulm

\section{Konrad Schütze}

Dr. med., Abteilung für Unfall-, Hand-, Plastische- und Wiederherstellungschirurgie, Universitätsklinikum Ulm

\section{Korrespondenzadresse}

\section{Dr. med. Peter Hinnerk Richter}

Abteilung für Unfall-, Hand-, Plastische- und

Wiederherstellungschirurgie

Universitätsklinikum Ulm

Albert-Einstein-Allee 23

$89081 \mathrm{Ulm}$

Tel.: 0731/500-54567

peter.richter@uniklinik-ulm.de

\section{Literatur}

[1] Zwingmann J, Hauschild O, Bode G et al. Malposition and revision rates of different imaging modalities for percutaneous iliosacral screw fixation following pelvic fractures: a systematic review and meta-analysis. Arch Orthop Trauma Surg 2013; 133: $1257-1265$

[2] Richter PH, Grafenberg A, Bodky M et al. Leistungsfähigkeit der Flat-Panel-Technology in 2-D/3-D. OP-Journal 2014; 30: 169-177

[3] Cowen AR, Kengyelics SM, Davies AG. Solid-state, flat-panel, digital radiography detectors and their physical imaging characteristics. Clin Radiol 2008; 63: 487-498

[4] Seibert JA. Flat-panel detectors: how much better are they? Pediatr Radiol 2006; 36 (Suppl. 2): S173-S181

[5] Krettek C, Gebhard F. [Development of intraoperative C-arm imaging in trauma surgery]. Unfallchirurg 2012; 115: 100 106

[6] Routt ML jr., Simonian PT, Mills WJ. Iliosacral screw fixation: early complications of the percutaneous technique. J Orthop Trauma 1997; 11: 584-589

[7] Raj S, Irani FG, Tay KH et al. C-arm cone beam computed tomography: a new tool in the interventional suite. Ann Acad Med Singapore 2013; 42: 585-592

[8] Shaw JC, Routt MLC jr., Gary JL. Intra-operative multi-dimensional fluoroscopy of guidepin placement prior to iliosacral screw fixation for posterior pelvic ring injuries and sacroiliac dislocation: an early case series. Int Orthop 2017; 41: 2171 2177
[9] Verma SK, Singh PK, Agrawal D et al. O-arm with navigation versus $C$-arm: a review of screw placement over 3 years at a major trauma center. Br J Neurosurg 2016; 30: 658-661

[10] Sullivan JP, Warme BA, Wolf BR. Use of an O-arm intraoperative computed tomography scanner for closed reduction of posterior sternoclavicular dislocations. J Shoulder Elbow Surg 2012; 21: e17-e20

[11] Hsu AR, Gross CE, Lee S. Intraoperative O-arm computed tomography evaluation of syndesmotic reduction: case report. Foot Ankle Int 2013; 34: 75375-9

[12] Richter PH, Yarboro S, Kraus M et al. One year orthopaedic trauma experience using an advanced interdisciplinary hybrid operating room. Injury 2015; 46 (Suppl. 4): S129-S134

[13] Richter PH, Gebhard F, Dehner C et al. Accuracy of computerassisted iliosacral screw placement using a hybrid operating room. Injury 2016; 47: 402-407

[14] Pieske O, Landersdorfer C, Trumm C et al. CT-guided sacroiliac percutaneous screw placement in unstable posterior pelvic ring injuries: accuracy of screw position, injury reduction and complications in 71 patients with 136 screws. Injury 2015; 46: 333-339

[15] Peng KT, Li YY, Hsu WH et al. Intraoperative computed tomography with integrated navigation in percutaneous iliosacral screwing. Injury 2013; 44: 203-208

[16] Barsa P, Frohlich R, Sercl M et al. The intraoperative portable CT scanner-based spinal navigation: a viable option for instrumentation in the region of cervico-thoracic junction. Eur Spine J 2016; 25: 1643-1650

[17] Hecht N, Kamphuis M, Czabanka M et al. Accuracy and workflow of navigated spinal instrumentation with the mobile AIRO ((R)) CT scanner. Eur Spine J 2016; 25: 716-723

[18] Czabanka M, Haemmerli ], Hecht $\mathrm{N}$ et al. Spinal navigation for posterior instrumentation of $\mathrm{C} 1-2$ instability using a mobile intraoperative CT scanner. J Neurosurg Spine 2017;27: 268275

[19] Tonetti J, Carrat L, Blendea S et al. Clinical results of percutaneous pelvic surgery. Computer assisted surgery using ultrasound compared to standard fluoroscopy. Comput Aided Surg 2001; 6: 204-211

[20] Hinsche AF, Giannoudis PV, Smith RM. Fluoroscopy-based multiplanar image guidance for insertion of sacroiliac screws. Clin Orthop Relat Res 2002; (395): 135-144

[21] Zwingmann J, Konrad G, Mehlhorn AT et al. Percutaneous iliosacral screw insertion: malpositioning and revision rate of screws with regards to application technique (navigated vs. Conventional). J Trauma 2010; 69: 1501-1506

[22] Ochs BG, Gonser C, Shiozawa T et al. Computer-assisted periacetabular screw placement: comparison of different fluoroscopy-based navigation procedures with conventional technique. Injury 2010;41(12):1297-305

[23] Kraus MD, Krischak G, Keppler P et al. Can computer-assisted surgery reduce the effective dose for spinal fusion and sacroiliac screw insertion? Clin Orthop Relat Res 2010; 468: 24192429

Bibliografie

DOI https://doi.org/10.1055/s-0044-100006 OP-JOURNAL 2018; 34: 18-24 @ C Georg Thieme Verlag KG Stuttgart · New York ISSN 0178-1715 